\title{
Node Penalty Evaluation in Multi Criteria Safety Route Problem: A ] Logic Approach
}

A FAHMY, M Rasmy, S Ali, M Ewees

\begin{abstract}
: Node penalty evaluation of a complex situation is an important yet difficult human decision making within the conceptual framework of fuzzy logic and approximating reasoning. The multi-criteria safety route problem is concerned with determining the safety route from an origin to a destination through a connected network, given the different criterion associated with each node of the network. In most cases routes are determined based on shortest distances least-cost or least-time. In our case in addition to the previous criterion other criteria such as elevation restricted areas type I and restricted areas type II are not considered as intelligent as they must be. To tackle the problem a new integration form took place between operations research and artificial intelligence. The statement of multiple criterion safety route problem with fuzzy coefficients will be presented. A simpler yet more effective methods are introduced to develop a fuzzy logic safety route model based on these different criteria of route parameters that converts fiizzified inputs to fuzzy output through fuzzy membership functions. A theorem [17] for finding an equivalent fuzzy single objective safety route problem which can be developed using the fuzzy inference of the FMSRP will he applied. Therefore we interpret fiizzy linear programming problems witii fuzzy coefficients in the multiple objectives fimctions as multiple fuzzy reasoning schemes where the antecedents of the scheme correspond to the coefficients of every decision variable $\mathrm{xj}$ in the all objective fimctions. Thereafter, we establish a Fuzzy inference model for the multiple objectives safety route problem. We perform a rule combination algorithm [18]. Then the solution process consists of three steps: first for every node we compute the fuzzy value of its coefficient in the equivalent objective function via sup-min convolution of the antecedents/objective fimctions. Second we compute their crisp values. Then an optimal solution of a large scale safety route problem will be obtained. Experimental work has been conducted to obtain the nodes information and observe the system behaviors which are interpreted by using systematic analysis as an aid to the design of fuzzy logic safety route modular. Simulation results show that fuzzy logic model with a rule combined and elimination method can reasonably get the safety route. The methodology presented in this
\end{abstract}


paper is expected to be applied to the mission planning systems to improve the node penalty evaluation process performance and maximize the safety of the routes. 